\title{
Optimum Design of Electrode Arrangements in a Multilayer Piezo-Composite Disk for Control of Thermal Stress *
}

\author{
Ahmed ELSAWAF**, Fumihiro ASHIDA*** and Sei-ichiro SAKATA*** \\ ${ }^{* *}$ Graduate School of Shimane University \\ 1060 Nishikawatsu-cho, Matsue, Shimane 690-8504, Japan \\ E-mail: ahmed99920032003@yahoo.com \\ ${ }^{* * *}$ Department of Electronic and Control Systems Engineering, Shimane University \\ 1060 Nishikawatsu-cho, Matsue, Shimane 690-8504, Japan \\ E-mail: ashida@ecs.shimane-u.ac.jp
}

\begin{abstract}
This paper deals with a control problem of a thermal stress in a composite circular disk consisting of a structural layer onto which multiple piezoceramic layers with concentrically arranged electrodes are bonded. When a heating temperature distribution acts on the bottom surface of the structural layer, the maximum thermal stress in the structural layer is minimized by applying appropriate voltages to the electrodes. The stepwise voltage applied to each electrode is obtained by solving a linear programming problem when constrains are imposed on stresses in the piezoceramic layers. In order to decrease design variables such as the layer thicknesses and the number and dimensions of electrodes, an efficient method for the optimum design is developed. The structure of the composite disk as well as the electrode arrangement on each piezoceramic layer has been optimally designed so that performance of the stress control is maximized. Finally, it is seen from numerical results that the maximum thermal stress in the structure layer is reduced by $38.3 \%$ which is $4.6 \%$ higher than that obtained in the previous paper.
\end{abstract}

Key words: Elasticity, Thermal Stress, Stress Control, Optimum Design, Piezoelectric Actuation, Multilayer Composite Disk, Smart Structure

\section{Introduction}

The utilization of piezoelectric-based smart structures technology gains increasing attention in engineering and industrial applications. Various electro-elastic problems relevant to piezoelectric-based smart structures have been treated. According to the extension of investigations, Rao and Sunar ${ }^{(1),(2)}$ and Irschik ${ }^{(3)}$ surveyed important articles on smart structures mainly operated under isothermal conditions, while Tauchert, et al. ${ }^{(4) \sim(6)}$ reviewed key papers concerning smart structures which sensed and controlled thermal responses.

Applications of piezoelectro-thermoelastic actuation have been focused on the control of a static deformation. For example, problems of controlling a thermal displacement distribution ${ }^{(7),(8)}$ were analyzed. However, there are a very few papers on a stress control problem.

The previous papers ${ }^{(9),(10)}$ demonstrated the possibility of controlling a thermal stress in a composite disk consisting of a structure layer and piezoceramic layers, when the structure 
layer surface was exposed to a heating temperature distribution. In the first paper ${ }^{(9)}$, assuming that a number of electrodes of a same width were arranged at an equal interval on each piezoceramic layer, voltages applied to the electrodes were determined by solving a nonlinear optimization problem, in order to minimize the maximum thermal stress in the structure layer. Since the solution strongly depended on the choice of initial optimization variables and easily got stuck in a local minimum then, it was difficult to obtain optimum solutions required for a design of the electrode arrangement. In the second paper ${ }^{(10)}$ which was an extension work of the first paper and discussed a case where electrodes of various widths were arranged at different intervals on each piezoceramic layer, the nonlinear optimization problem was transformed into a linear programming problem by introducing new optimization variables and thus all optimum solutions were successfully obtained. However, the analyses in both papers were based on the assumption that a same electrode arrangement was attached to every piezoceramic layer. In addition, the effect of the layer thicknesses on performance of the stress control was not examined. For more successful and efficient control of a thermal stress, a further research is needed.

The present paper extends the previous works ${ }^{(9),(10)}$ to an optimum design for the structure of the composite disk and electrode arrangements. When a different electrode arrangement is attached to each piezoceramic layer, voltages applied to the electrodes are determined by solving a linear programming program subject to constraints on stresses in the piezoceramic layers. The thicknesses of the piezoceramic layers as well as the width and interval of each electrode have been determined in order to demonstrate the function of stress control to the fullest extent possible. For completion of the optimum design, the linear programming problem must be repeatedly solved under different conditions for the thousandth time, and therefore an efficient method for the optimum design is developed. Finally, numerical results obtained for a composite disk constructed of a CFRP layer and cadmium selenide layers are shown in tables and figures. The numerical results illustrate how much influence the thickness of each piezoceramic layer as well as the electrode arrangement on each piezoceramic layer exerts upon suppression of the maximum thermal stress in the structural layer.

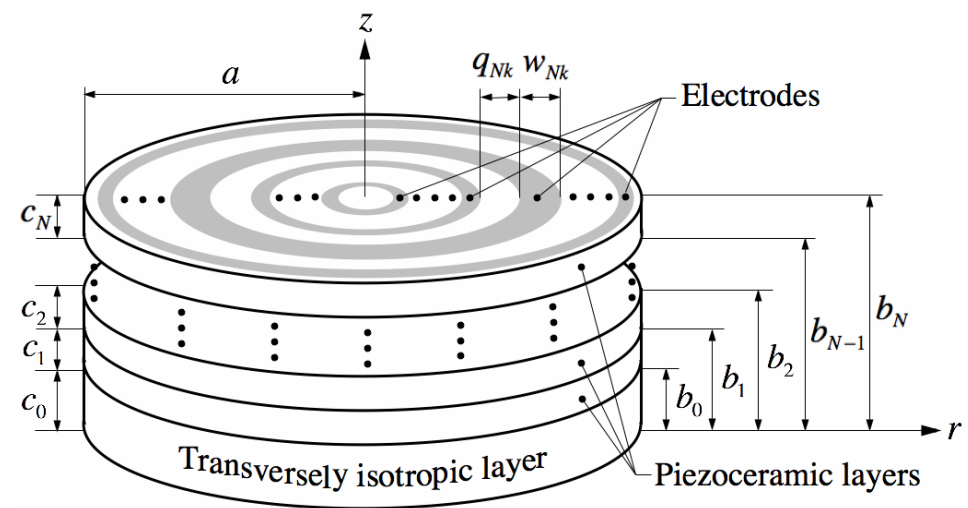

Fig. 1 Geometry of a multilayer composite disk

\section{Presentation of Problem and Analysis}

Let us consider a multilayer composite disk consisting of a transversely isotropic structure layer onto which piezoceramic layers of crystal class $6 \mathrm{~mm}$ are perfectly bonded as shown in Fig. 1. It is assumed that a number of electrodes are arranged concentrically on the upper surface of each piezoceramic layer. The constituent layers are numbered 0 to $N$ from the bottom and the electrodes on the $i$ th layer are numbered 1 to $M_{i}$ from the center. The width of the $k$ th electrode and the interval between the $k$ th and $(k-1)$ th 
electrodes on the $i$ th layer are represented by $w_{i k}$ and $q_{i k}$, respectively.

\subsection{Thermo-elastic Response}

Let us consider a thermoelastic problem of the composite disk, when a heating temperature distribution $T_{c} f(r)$ acts on the bottom surface, heat convection occurs over the top surface, and the cylindrical surface is thermally insulated. The thermal boundary and interference conditions are expressed as:

$$
\begin{aligned}
& T_{0, z}-h_{0} T_{0}=-h_{0} T_{c} f(r) \text { on } z=0 \\
& T_{i}=T_{i+1},-\lambda_{i z} T_{i, z}=-\lambda_{(i+1) z} T_{(i+1), z} \text { on } z=b_{i} \quad(i=0 \sim N-1) \\
& T_{N, z}-h_{N} T_{N}=0 \text { on } z=b_{N} \\
& T_{i, r}=0 \text { on } r=a(i=0 \sim N)
\end{aligned}
$$

where $T_{i}$ is the temperature rise in the $i$ th layer, $T_{c}$ is a constant temperature, $h_{i}$ are coefficients of relative heat transfer, $\lambda_{i z}$ are coefficients of thermal conductivity, and $T_{i, z}=\partial T_{i} / \partial z$.

It is also assumed that the cylindrical surface of the composite disk is smoothly constrained against radial deformation, the top and bottom surfaces are stress-free, and the cylindrical edge of the bottom surface is simply supported. In this case the elastic boundary and interference conditions are given by

$$
\begin{aligned}
& \sigma_{0 z z}^{T}=\sigma_{0 r z}^{T}=0 \text { on } z=0 \\
& u_{i r}^{T}=u_{(i+1) r}^{T}, u_{i z}^{T}=u_{(i+1) z}^{T}, \sigma_{i z z}^{T}=\sigma_{(i+1) z z}^{T}, \sigma_{i r z}^{T}=\sigma_{(i+1) r z}^{T} \text { on } z=b_{i} \quad(i=0 \sim N-1) \\
& \sigma_{N z z}^{T}=\sigma_{N z}^{T}=0 \text { on } z=b_{N} \\
& u_{i r}^{T}=0, \sigma_{i r z}^{T}=0 \text { on } r=a(i=0 \sim N) \\
& u_{0 z}^{T}=0 \text { at } z=0, \quad r=a
\end{aligned}
$$

where $u_{i j}^{T}$ are displacements, $\sigma_{i j}^{T}$ are stresses, and symbols with the superscript $T$ hereafter denote response quantities induced by the thermal load.

Assuming that the upper and cylindrical surfaces of all piezoceramic layers are free of electric charge and the lower surface of each piezoceramic layer is electrically grounded, the electric boundary conditions are given by

$$
\begin{aligned}
& \Phi_{i}^{T}=0 \text { on } z=b_{i-1} \quad(i=1 \sim N) \\
& D_{i z}^{T}=0 \text { on } z=b_{i} \quad(i=1 \sim N) \\
& D_{i r}^{T}=0 \text { on } r=a \quad(i=1 \sim N)
\end{aligned}
$$

where $\Phi_{i}^{T}$ are electric potentials and $D_{i}^{T}$ are dielectric flux densities.

The functions representing the temperature rise, displacements, and stresses in the structural layer as well as representing the temperature rise, displacements, stresses, electric potential, and dielectric flux densities in every piezoceramic layer, which satisfy their governing equations, constitutive equations, and boundary conditions, have been obtained by employing the potential methods ${ }^{(10)}$. Therefore, they are omitted here for brevity.

\subsection{Electro-elastic Response}

It is assumed that a stepwise voltage is applied to each electrode on the $i$ th piezoceramic layer as:

$$
\Phi_{i}^{E}=\sum_{k=1}^{M_{i}} V_{i k}\left\{H\left(r-r_{i k}\right)-H\left(r-r_{i k}-w_{i k}\right)\right\} \text { on } z=b_{i} \quad(i=0 \sim N)
$$


where $V_{i k}$ is the voltage applied to the $k$ th electrode in the $i$ th layer (i.e., $(i-k)$ th electrode), $H(r)$ is Heaviside's unit step function, $r_{i k}$ is the inner radius of the $(i-k)$ th electrode, and symbols with the superscript $E$ denote response quantities due to the electric load. The boundary conditions given in Eqs. (5) (10) and (12) are still applicable for this situation, but the superscript must be changed from $T$ to $E$.

In order to analyze this problem effectively, a case where the unit voltage $V_{u}$ is applied to only the $(n-m)$ th electrode is considered. The discrete displacements $\left(\Delta u_{i j}^{E}\right)_{n m}$, stresses $\left(\Delta \sigma_{i j k}^{E}\right)_{n m}$, electric potentials $\left(\Delta \Phi_{i}^{E}\right)_{n m}$, and dielectric flux densities $\left(\Delta D_{i j}^{E}\right)_{n m}$ induced by the unit voltage have been obtained by employing the potential function methods ${ }^{(10)}$.

When a voltage of arbitrary magnitude $V_{n m}$ is applied to the $(n-m)$ th electrode, it is expressed by

$$
V_{n m}=P_{n m} V_{u}
$$

where $P_{n m}$ is the magnification factor of the applied voltage. In the case where an arbitrary voltage is applied to every electrode, the response quantities are given by

$$
\left\{u_{i j}^{E}, \sigma_{i j k}^{E}, \Phi_{i}^{E}, D_{i j}^{E}\right\}=\sum_{n=1}^{N} \sum_{m=1}^{M_{n}} P_{n m}\left\{\left(\Delta u_{i j}^{E}\right)_{n m},\left(\Delta \sigma_{i j k}^{E}\right)_{n m},\left(\Delta \Phi_{i}^{E}\right)_{n m},\left(\Delta D_{i j}^{E}\right)_{n m}\right\}
$$

\subsection{Resultant Responses}

The resultant displacements, stresses, electric potentials, and dielectric flux densities due to both thermal and electrical loads are obtained by superimposing the responses due to each load as:

$$
u_{i j}=u_{i j}^{T}+u_{i j}^{E}, \sigma_{i j k}=\sigma_{i j k}^{T}+\sigma_{i j k}^{E}, \Phi_{i}=\Phi_{i}^{T}+\Phi_{i}^{E}, D_{i j}=D_{i j}^{T}+D_{i j}^{E}
$$

where the symbols without a superscript represent the resultant responses.

\section{Linear Programming Problem}

Let us determine the voltages $V_{i k}$ applied to all electrodes so that the maximum thermal stress in the structure layer is minimized subject to constraints on the stresses in the piezoceramic layers. This optimization problem is defined by

$$
\left.\begin{array}{ll}
\text { find } & \boldsymbol{X}=\left(P_{11}, P_{12}, \ldots \ldots . P_{N M_{N}}, R_{s}\right)^{\mathrm{T}} \\
\text { to minimize } & f_{o b j}(\boldsymbol{X})=R_{s}\left|\sigma_{0 \max }^{T}\right| \\
\text { subject to } & \left\{\left|\sigma_{0 r r}\right|,\left|\sigma_{0 \theta \theta}\right|,\left|\sigma_{0 z z}\right|,\left|\sigma_{0 r z}\right|\right\} \leq R_{s}\left|\sigma_{0 \max }^{T}\right|, \\
& \sigma_{p c}^{A} \leq\left\{\sigma_{p r r}, \sigma_{p \theta \theta}, \sigma_{p z z}\right\} \leq \sigma_{p t}^{A}, \\
& -\sigma_{p s}^{A} \leq \sigma_{p r z} \leq \sigma_{p s}^{A}
\end{array}\right\}
$$

where ()$^{\mathrm{T}}$ denotes a transposed matrix, $f_{o b j}(X)$ is the object function, $\sigma_{0 \max }^{T}$ is the maximum thermal stress in the structure layer, $\sigma_{p r r}, \sigma_{p \theta \theta}, \sigma_{p z z}$, and $\sigma_{p r z}$ are the maximum stress components in the piezoceramic layers, $\sigma_{p t}^{A}, \sigma_{p c}^{A}$, and $\sigma_{p s}^{A}$ are the allowable tensile, compressive, and shear stresses, and $R_{s}$ is the optimization variable defined as: 


$$
R_{s}=\frac{\left|\sigma_{0 \max }\right|}{\left|\sigma_{0 \max }^{T}\right|}
$$

where $\sigma_{0 \max }$ is the maximum stress in the structure layer after applying the voltages.

In order to assess the performance of the stress control, the ratio $R$ evaluating the suppression of the maximum thermal stress in the structure layer is introduced.

$$
R=\left(1-R_{s}\right) \times 100[\%]
$$

\section{Numerical Results}

For convenience in presentation of numerical results, the following dimensionless quantities are introduced:

$$
\left(\bar{r}, \bar{z}, \overline{c_{i}}, \overline{b_{i}}, \bar{w}_{i k}, \bar{q}_{i k}\right)=\frac{\left(r, z, c_{i}, b_{i}, w_{i k}, q_{i k}\right)}{a}, \overline{V_{i k}}=\frac{\left|d_{1}\right| V_{i k}}{a \tilde{\alpha}_{r} T_{c}}, \bar{\sigma}_{i j k}=\frac{\sigma_{i j k}}{\tilde{\alpha}_{r} Y_{r} T_{c}}, B_{i}=a h_{i}
$$

where $d_{1}$ is the piezoelectric coefficient, $\tilde{\alpha}_{r}$ and $\tilde{Y_{r}}$ are the coefficient of linear thermal expansion and Young's modulus of the structural layer, and $B_{i}$ are Biot's numbers.

The structural layer is considered to be carbon fiber reinforced plastic (CFRP), whereas the piezoceramic layers are considered to be cadmium selenide. The material constants are given in the previous paper ${ }^{(9)}$.

The heating temperature acting on the bottom surface of the composite disk is considered to have the following distribution:

$$
f(\bar{r})=H\left(\overline{r_{o}}-\bar{r}\right)\left(1-2 \frac{\bar{r}^{2}}{\bar{r}_{o}^{2}}+\frac{\bar{r}^{4}}{\bar{r}_{o}^{4}}\right)
$$

where $\bar{r}_{o}$ is the radius of the heating region and here it is taken to be $\bar{r}_{o}=0.5$. In order to improve the convergence of infinite series contained in the analytical solution, the unit step function is approximated as:

$$
H\left(\bar{r}-\bar{r}_{i k}+\bar{w}_{i k}\right)-H\left(\bar{r}-\bar{r}_{i k}\right)=\frac{1}{\left[1+\exp \left\{-s\left(\bar{r}-\bar{r}_{i k}+\bar{w}_{i k}\right)\right\}\right]\left[1+\exp \left\{s\left(\bar{r}-\bar{r}_{i k}\right)\right\}\right]}
$$

where $s$ is a parameter which governs the accuracy of the approximation and is taken to be $s=200$. It is assumed that the thickness of the structure layer is $\overline{c_{0}}=0.002$ and Biot's numbers are $B_{0}=1$ and $B_{N}=0.1$. The allowable stresses in the piezoceramic layers are assumed to be $\bar{\sigma}_{p t}^{A}=0.004, \quad \bar{\sigma}_{p c}^{A}=-0.04$, and $\bar{\sigma}_{p s}^{A}=0.002$.

\subsection{Determination of Optimum Layer Thicknesses}

Let us determine the optimum thickness of each piezoceramic layer so that the suppression ratio of the maximum thermal stress in the structural layer is maximized. The linear optimization problem given in Eq. (17) has been solved for a composite disk with two or three piezoceramic layers of various thicknesses when a same electrode arrangement is attached to every piezoceramic layer, namely $q_{i k}=q_{k}, w_{i k}=w_{k}$. In this case the following electrode arrangements which were found to be the best in the previous paper ${ }^{(10)}$ are used.

$$
\bar{q}_{1}=0, \bar{w}_{1}=0.4, \bar{q}_{2}=0.1, \bar{w}_{2}=0.175, \bar{q}_{3}=0.025, \bar{w}_{3}=0.3 \text { for } N=2
$$




$$
\bar{q}_{1}=0, \bar{w}_{1}=0.4, \bar{q}_{2}=0.1, \bar{w}_{2}=0.125, \bar{q}_{3}=0.025, \bar{w}_{3}=0.35 \text { for } N=3
$$

Table 1 shows the effect of the total thickness of the piezoceramic layers on the suppression ratio of the maximum thermal stress for the case of two and three piezoceramic layers of an equal thickness, namely $\overline{c_{i}}=\bar{c}$. It is seen from Table 1 that the maximum suppression ratio is obtained when the total thickness is taken to be 0.035 for the case of two piezoceramic layers and 0.025 for the case of three piezoceramic layers, respectively.

Table 2 shows how the thickness of each piezoceramic layer exerts an influence upon the suppression ratio of the maximum thermal stress for the case of two and three piezoceramic layers of different thicknesses, when the total thickness of the piezoceramic layers is taken to be 0.035 for the case of two piezoceramic layers and 0.025 for the case of three piezoceramic layers based on the numerical results in Table 1. From Tables 1 and 2, the highest suppression ratio is found to be obtained when the thicknesses of the two piezoceramic layers are $\overline{c_{1}}=\bar{c}_{2}=0.0175$ and those of the three piezoceramic layers are $\overline{c_{1}}=0.008, \bar{c}_{2}=0.009$, and $\overline{c_{3}}=0.008$, respectively. However, in the case of three piezoceramic layers, a close second of the suppression ratios is obtained when the thicknesses are equal, namely $\overline{c_{1}}=\overline{c_{2}}=\overline{c_{3}}=0.00833$. Therefore, in this study, the composite disk with multiple piezoceramic layers of an equal thickness is considered to be an optimum structure.

Table 1 Effect of the total thickness of piezoceramic layers on the suppression ratio for the case of two and three piezoceramic layers of equal thickness $\left(\overline{c_{i}}=\bar{c}\right)$

\begin{tabular}{|l|l|l|}
\hline \multirow{2}{*}{$\sum_{i=1}^{N} \overline{c_{i}}$} & \multicolumn{2}{|c|}{$R[\%]$} \\
\cline { 2 - 3 } & $N=2$ & $N=3$ \\
\hline 0.015 & 24.00 & 25.10 \\
\hline 0.020 & 29.87 & 33.67 \\
\hline 0.025 & 32.31 & 35.61 \\
\hline 0.030 & 33.19 & 35.02 \\
\hline 0.035 & 33.58 & 34.13 \\
\hline 0.040 & 32.80 & 33.20 \\
\hline 0.045 & 32.07 & 31.70 \\
\hline
\end{tabular}

Table 2 Effect of the thickness of each piezoceramic layer on the suppression ratio for the case of two and three piezoceramic layers of different thicknesses

\begin{tabular}{|c|c|c|c|c|c|c|}
\hline \multicolumn{3}{|c|}{$N=2, \sum_{i=1}^{N} \overline{c_{i}}=0.035$} & \multicolumn{4}{c|}{$N=3, \sum_{i=1}^{N} \overline{c_{i}}=0.025$} \\
\hline$\overline{c_{1}}$ & $\overline{c_{2}}$ & $R[\%]$ & $\overline{c_{1}}$ & $\overline{c_{2}}$ & $\overline{c_{3}}$ & $R[\%]$ \\
\hline 0.005 & 0.030 & 17.99 & 0.005 & 0.005 & 0.015 & 31.04 \\
\hline 0.0075 & 0.0275 & 20.78 & 0.005 & 0.010 & 0.010 & 35.43 \\
\hline 0.010 & 0.025 & 24.93 & 0.005 & 0.015 & 0.005 & 34.89 \\
\hline 0.015 & 0.020 & 30.61 & 0.008 & 0.009 & 0.008 & 35.63 \\
\hline 0.020 & 0.015 & 33.18 & 0.010 & 0.005 & 0.010 & 35.25 \\
\hline 0.025 & 0.010 & 30.99 & 0.010 & 0.010 & 0.005 & 35.04 \\
\hline 0.030 & 0.005 & 27.06 & 0.015 & 0.005 & 0.005 & 34.38 \\
\hline
\end{tabular}

\subsection{Determination of Optimum Electrode Arrangements}

The linear optimization problem given in Eq. (17) has been solved for various numbers, widths, and intervals of the electrodes, when a different electrode arrangement is attached to each piezoceramic layer. The optimum electrode arrangement on the $i$ th piezoceramic layer with $M_{i}$ electrodes is then determined as follows: 
(1) Step-1: Solve the linear programming problem given in Eq. (17) for all admissible dimensions of the electrodes which satisfy the following conditions.

$$
\sum_{k=1}^{M_{i}}\left(\bar{w}_{i k}+\bar{q}_{i k}\right)=1,\left(\bar{w}_{i k}, \bar{q}_{i k}\right)=(0.05,0.1,0.15, \ldots ., 0.4)
$$

(2) Step-2: Select electrode dimensions $\left(\bar{w}_{i k}, \bar{q}_{i k}\right)_{\text {S1-m }}$ which produce 15 high suppression ratios from the numerical results obtained in Step-1.

(3) Step-3: Letting the electrode dimensions be

$$
\left(\bar{w}_{i k}, \bar{q}_{i k}\right)_{\mathrm{S} 2-\mathrm{m} \pm}=\left(\bar{w}_{i k}, \bar{q}_{i k}\right)_{\mathrm{S} 1-\mathrm{m}} \pm 0.025
$$

solve the linear programming problem repeatedly for all sets of electrode arrangements $\left(\bar{w}_{i k}, \bar{q}_{i k}\right)_{\mathrm{S} 2-\mathrm{m} \pm}$.

(4) Step-4: Select one electrode arrangement which produces the highest suppression from the numerical results obtained in Step-1 and Step-3.

For a multiple composite disk, optimum thicknesses of the piezoceramic layers were determined for the case of the best electrode arrangements derived in the previous paper ${ }^{(10)}$ as shown in Section 4.1 and then optimum electrode arrangements were designed using the determined layer thicknesses. Since the suppression ratio little changed when the thicknesses of the piezoceramic layers were redesigned utilizing the designed electrode arrangements, layer thicknesses and electrode arrangements were respectively designed only once. Now, for a two-layer composite disk, both designs were interdependent with each other, so that the thickness of the piezoceramic layer and the electrode arrangement were respectively designed repeatedly using Step-1 to Step-4 until a maximum suppression ratio was obtained.

\subsubsection{Numerical results for the case of three electrodes}

For the case of one piezoceramic layer with three electrodes, the layer thickness and optimum electrode arrangement have been determined, repeating the design of the layer thickness and electrode arrangement three times. The determined electrode arrangement is applied to the case of two and three piezoceramic layers with a same electrode arrangement. The obtained numerical results are shown in Table 3. The suppression ratio is found to increase with increasing the piezoceramic layers. According to the numerical results obtained in the previous paper ${ }^{(10)}$ when the total thickness of the piezoceramic layers was taken to be 0.02 , the maximum suppression ratios for the case of one, two, and three piezoceramic layers are $20.3 \%, 29.8 \%$, and $33.6 \%$, respectively.

Table 3 Numerical results for a composite disk with a same arrangement of three electrodes $\left(\overline{c_{i}}=\bar{c}, M_{i}=M, \bar{w}_{i k}=\bar{w}_{k}, \bar{q}_{i k}=\bar{q}_{k}\right)$

\begin{tabular}{|c|c|c|c|c|c|c|c|c|c|}
\hline$N$ & $\sum_{i=1}^{N} \bar{c}_{i}$ & $M$ & $\bar{w}_{1}$ & $\bar{w}_{2}$ & $\bar{w}_{3}$ & $\bar{q}_{1}$ & $\bar{q}_{2}$ & $\bar{q}_{3}$ & $R[\%]$ \\
\hline 1 & 0.029 & 3 & 0.325 & 0.050 & 0.375 & 0.000 & 0.025 & 0.225 & 24.21 \\
\hline 2 & 0.035 & 3 & 0.325 & 0.050 & 0.375 & 0.000 & 0.025 & 0.225 & 34.33 \\
\hline 3 & 0.025 & 3 & 0.325 & 0.050 & 0.375 & 0.000 & 0.025 & 0.225 & 36.20 \\
\hline
\end{tabular}

Table 4 Numerical results for a composite disk with a same arrangement of three electrodes obtained from redesigns $\left(\overline{c_{i}}=\bar{c}, M_{i}=M, \bar{w}_{i k}=\bar{w}_{k}, \bar{q}_{i k}=\bar{q}_{k}\right)$

\begin{tabular}{|c|c|c|c|c|c|c|c|c|c|}
\hline$N$ & $\sum_{i=1}^{N} \bar{c}_{i}$ & $M$ & $\bar{w}_{1}$ & $\bar{w}_{2}$ & $\bar{w}_{3}$ & $\bar{q}_{1}$ & $\bar{q}_{2}$ & $\bar{q}_{3}$ & $R[\%]$ \\
\hline 2 & 0.035 & 3 & 0.325 & 0.100 & 0.300 & 0.000 & 0.025 & 0.250 & 34.96 \\
\hline 3 & 0.025 & 3 & 0.375 & 0.075 & 0.350 & 0.000 & 0.025 & 0.175 & 36.25 \\
\hline
\end{tabular}


Table 5 Numerical results for a composite disk with different arrangements of three electrodes redesigned based on results in Table $3\left(\overline{c_{i}}=\bar{c}, M_{i}=M\right)$

\begin{tabular}{|c|c|c|c|c|c|c|c|c|c|c|}
\hline$N$ & $i$ & $\bar{c}$ & $M$ & $\bar{w}_{i 1}$ & $\bar{w}_{i 2}$ & $\bar{w}_{i 3}$ & $\bar{q}_{i 1}$ & $\bar{q}_{i 2}$ & $\bar{q}_{i 3}$ & $R[\%]$ \\
\hline \multirow{2}{*}{2} & 1 & 0.0175 & 3 & 0.325 & 0.050 & 0.375 & 0.000 & 0.025 & 0.225 & \multirow{2}{*}{36.74} \\
\cline { 2 - 11 } & 2 & 0.0175 & 3 & 0.125 & 0.150 & 0.225 & 0.000 & 0.350 & 0.150 & \\
\hline \multirow{3}{*}{3} & 1 & 0.00833 & 3 & 0.325 & 0.050 & 0.375 & 0.000 & 0.025 & 0.225 & \\
& 2 & 0.00833 & 3 & 0.325 & 0.050 & 0.375 & 0.000 & 0.025 & 0.225 & \multirow{3}{*}{37.65} \\
\cline { 2 - 10 } & 3 & 0.00833 & 3 & 0.125 & 0.125 & 0.250 & 0.000 & 0.200 & 0.300 & \\
\hline
\end{tabular}

Table 6 Numerical results for a composite disk with different arrangements of three electrodes redesigned based on results in Table $4\left(\overline{c_{i}}=\bar{c}, M_{i}=M\right)$

\begin{tabular}{|c|c|c|c|c|c|c|c|c|c|c|}
\hline$N$ & $i$ & $\bar{c}$ & $M$ & $\bar{w}_{i 1}$ & $\bar{w}_{i 2}$ & $\bar{w}_{i 3}$ & $\bar{q}_{i 1}$ & $\bar{q}_{i 2}$ & $\bar{q}_{i 3}$ & $R[\%]$ \\
\hline \multirow{2}{*}{2} & 1 & 0.0175 & 3 & 0.325 & 0.100 & 0.300 & 0.000 & 0.025 & 0.250 & \multirow{3}{*}{35.88} \\
\cline { 2 - 11 } & 2 & 0.0175 & 3 & 0.300 & 0.100 & 0.050 & 0.000 & 0.225 & 0.325 & \\
\hline \multirow{3}{*}{3} & 1 & 0.00833 & 3 & 0.375 & 0.075 & 0.350 & 0.000 & 0.025 & 0.175 & \\
\cline { 2 - 10 } & 2 & 0.00833 & 3 & 0.375 & 0.075 & 0.350 & 0.000 & 0.025 & 0.175 & \multirow{3}{*}{37.52} \\
\cline { 2 - 9 } & 3 & 0.00833 & 3 & 0.100 & 0.175 & 0.250 & 0.000 & 0.075 & 0.400 & \\
\hline
\end{tabular}

Table 7 Numerical results for a four-layer composite disk with different arrangements of three electrodes redesigned based on results in Table $5\left(\overline{c_{i}}=\bar{c}, M_{i}=M\right)$

\begin{tabular}{|c|c|c|c|c|c|c|c|c|c|c|}
\hline$N$ & $i$ & $\bar{c}$ & $M$ & $\bar{w}_{i 1}$ & $\bar{w}_{i 2}$ & $\bar{w}_{i 3}$ & $\bar{q}_{i 1}$ & $\bar{q}_{i 2}$ & $\bar{q}_{i 3}$ & $R[\%]$ \\
\hline \multirow{4}{*}{3} & 1 & 0.00833 & 3 & 0.325 & 0.050 & 0.375 & 0.000 & 0.025 & 0.225 & \\
\cline { 2 - 11 } & 2 & 0.00833 & 3 & 0.325 & 0.050 & 0.400 & 0.000 & 0.025 & 0.200 & \multirow{3}{*}{37.80} \\
\cline { 2 - 11 } & 3 & 0.00833 & 3 & 0.125 & 0.125 & 0.250 & 0.000 & 0.200 & 0.300 & \\
\hline
\end{tabular}

In the case of two and three piezoceramic layers with a same electrode arrangement, the optimum electrode arrangements have been redesigned and the obtained numerical results are shown in Table 4. It is seen from Tables 3 and 4 that slightly greater suppression ratios are obtained when the electrode arrangements are optimally redesigned.

Based on the numerical results shown in Table 3, only the electrode arrangement on the top piezoceramic layer has been optimally redesigned for the case of two and three piezoceramic layers, in which case the electrode arrangements on the other piezoceramic layers are the same to those given in Table 3. The obtained numerical results are shown in Table 5. It is seen that the suppression ratios in Table 5 are about $1.5 \%$ greater than those in Table 4.

Applying the similar fashion, the electrode arrangement on the top piezoelectric layer has been optimally redesigned for the case of two and three piezoceramic layers, when the electrode arrangements on the other piezoceramic layers are the same to those given in Table 4. Table 6 shows the obtained numerical results. Comparing both results in Tables 5 and 6, the suppression ratios shown in Table 5 are somewhat greater than those given in Table 6.

For a composite disk with three piezoceramic layers, the electrode arrangement of the second piezoceramic layer is redesigned when the electrode arrangements on the first and third piezoceramic layers are the same to those given in Table 5. Table 7 shows the obtained numerical results. From the numerical results shown in Tables 5 and 7, the suppression ratio is found to increase from $37.65 \%$ to $37.80 \%$, but they are almost equal.

\subsubsection{Numerical results for the case of multiple electrodes}

The suppression ratio of the maximum thermal stress in the structure layer is examined 
for the case of two piezoceramic layers with multiple electrodes. Table 8 shows the numerical results obtained for various electrode arrangements. In this table, the arrangements of the electrodes assigned to the row of "Designed electrodes" are optimally designed, while the arrangements of the other electrodes assigned to the row of "Fixed electrodes" are the same to those given in Table 5. In addition, a case where two electrodes are assigned to the row of "Designed electrodes" means that the same electrode arrangement is attached to both piezoceramic layers. It is seen from Tables 5 and 8 that the suppression ratio somewhat increases according to only the electrode number of the top piezoceramic layer, namely $M_{2}$.

The similar examination has been carried out for a composite disk with three piezoceramic layers. Table 9 shows the numerical results obtained for various electrode arrangements. In this table, the arrangements of the electrodes assigned to the row of "Fixed electrodes" are the same to those given in Table 7. Tables 7 and 9 bring the result, which is similar to the case in Tables 5 and 8, that the suppression ratio slightly increases with increment of only the electrodes on the top piezoceramic layer, namely $M_{3}$.

Table 8 Numerical results for the case of two piezoceramic layers with different arrangements of multiple electrodes designed based on results in Table $5\left(\overline{c_{i}}=\bar{c}\right)$

\begin{tabular}{|c|c|c|c|c|c|c|}
\hline \multirow{2}{*}{$N$} & $\bar{c}$ & \multicolumn{2}{|c|}{ Fixed electrodes } & \multicolumn{2}{|c|}{ Designed electrodes } & \multirow{2}{*}{$R[\%]$} \\
\cline { 3 - 6 } & & $M_{1}$ & $M_{2}$ & $M_{1}$ & $M_{2}$ & \\
\hline \multirow{4}{*}{2} & 0.0175 & 3 & - & - & 4 & 37.25 \\
\cline { 2 - 6 } & 0.0175 & 3 & - & - & 5 & 37.51 \\
\cline { 2 - 6 } & 0.0175 & 3 & - & - & 6 & 38.07 \\
\cline { 2 - 6 } & 0.0175 & - & 3 & 4 & - & 34.97 \\
\cline { 2 - 6 } & 0.0175 & - & - & \multicolumn{2}{|c}{4} & 32.71 \\
\cline { 2 - 6 } & 0.0175 & - & - & \multicolumn{2}{|c}{5} & 31.36 \\
\hline
\end{tabular}

Table 9 Numerical results for the case of three piezoceramic layers with various arrangements of multiple electrodes designed based on results in Table $7\left(\overline{c_{i}}=\bar{c}\right)$

\begin{tabular}{|c|c|c|c|c|c|c|c|c|}
\hline \multirow{3}{*}{$N$} & \multirow{c}{*}{$\bar{c}$} & \multicolumn{3}{|c|}{ Fixed electrodes } & \multicolumn{3}{c|}{ Designed electrodes } & \multirow{2}{*}{$R[\%]$} \\
\cline { 3 - 9 } & & $M_{1}$ & $M_{2}$ & $M_{3}$ & $M_{1}$ & $M_{2}$ & $M_{3}$ & \\
\hline \multirow{4}{*}{3} & 0.00833 & 3 & 3 & - & - & - & 4 & 38.12 \\
\cline { 2 - 9 } & 0.00833 & 3 & 3 & - & - & - & 5 & 38.12 \\
\cline { 2 - 9 } & 0.00833 & 3 & 3 & - & - & - & 6 & 38.22 \\
\cline { 2 - 9 } & 0.00833 & 3 & - & 3 & - & 4 & - & 35.50 \\
\cline { 2 - 9 } & 0.00833 & 3 & - & - & - & 4 & 34.69 \\
\cline { 2 - 9 } & 0.00833 & 3 & - & - & - & 5 & 34.19 \\
\hline
\end{tabular}

\subsection{Efficient Method for Optimum Design of Electrode Arrangements}

According to the numerical results discussed in the foregoing section, it is easy to suppose that the optimum design of the layer thicknesses and electrode arrangements needs huge number of data. For example, the optimum electrode arrangement even for each case in Table 4 was selected from among about 5,000 data sets $\left(\bar{w}_{i k}, \bar{q}_{i k}\right)$. Therefore, an efficient method for the optimum design is required in order to decrease design variables.

Now, the electrode arrangements which produce 10 high suppression ratios are selected from among the data used for determining the optimum electrode arrangement in the case of two piezoelectric layers shown in Table 5, and the configurations are illustrated in Fig. 2.

From Tables 5, 6, and 8, the numerical results for the case of two piezoceramic layers show that the highest suppression ratio is $R=38.07 \%$ when six electrodes are arranged on 
the top piezoceramic layer, but the suppression ratio is $R=36.74 \%$ in the case of three electrodes on the top piezoceramic layer. The difference between both suppression ratios is not more than about $1.3 \%$ and thus the number of electrodes could be limited to three. The suppression ratio $R=36.74 \%$ is obtained when only the electrode arrangement on the top piezoceramic layer is redesigned, in which case the optimum arrangement in the case of one piezoceramic layer is attached to the other piezoceramic layer. Applying this direction to the case of three piezoceramic layers, the suppression ratio $R=37.65 \%$ is obtained as shown in Table 5, but the highest suppression ratio is $R=38.22 \%$ when six electrodes are arranged on the top piezoceramic layer as shown in Table 9.

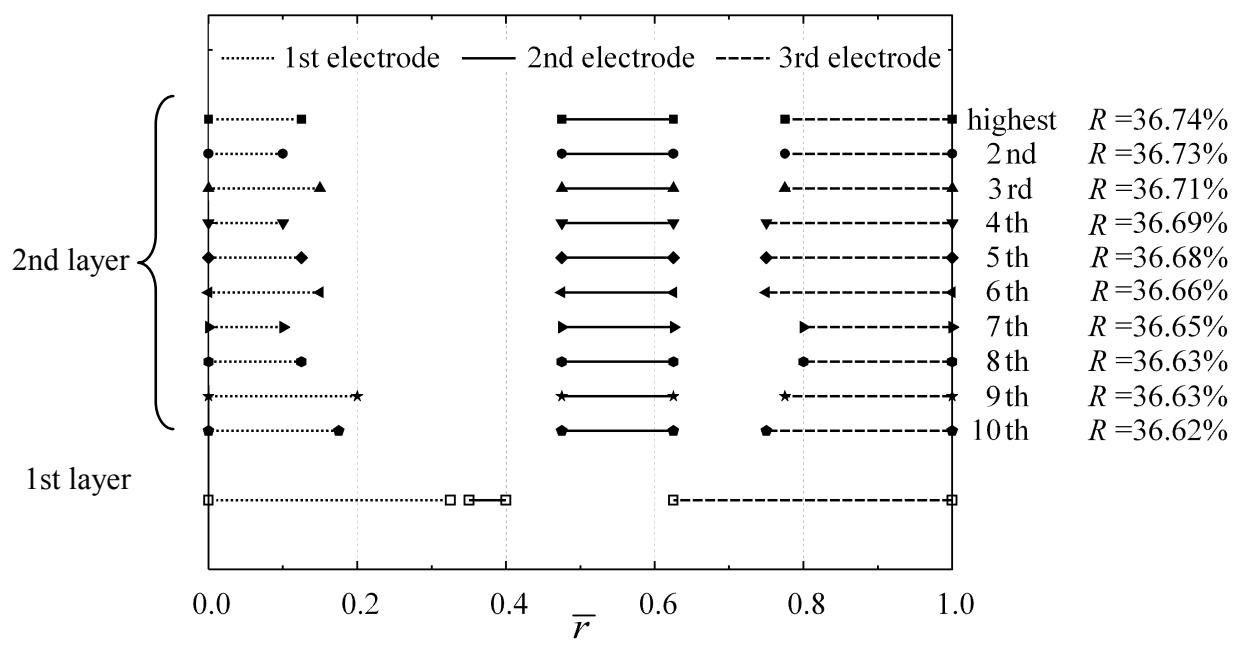

Fig. 2 Ten best electrode arrangements for the case of two piezoceramic layers with three electrodes each

Based on the above arguments, the direction for designing only the arrangement of three electrodes on the top piezoceramic layer is to be required. Examination into the results illustrated in Fig. 2 leads to the relationships of the electrode widths and intervals between the first and top piezoceramic layers as follows:

$$
\begin{aligned}
& \frac{\bar{w}_{11}}{2}-0.05 \leq \bar{w}_{N 1} \leq \frac{\bar{w}_{11}}{2}+0.05 \\
& \frac{\bar{q}_{12}+\bar{w}_{12}+\bar{q}_{13}}{2}-0.025 \leq \bar{w}_{N 2} \leq \frac{\bar{q}_{12}+\bar{w}_{12}+\bar{q}_{13}}{2}+0.075, \\
& \frac{\bar{w}_{13}}{2} \leq \bar{w}_{N 3} \leq \frac{\bar{w}_{13}}{2}+0.05
\end{aligned}
$$

where the increments of $\bar{w}_{N k}$ and $\bar{q}_{N k}$ are taken to be 0.025 .

The following technique for design of the layer thicknesses as well as of electrode arrangements can be proposed.

(1) Step-1: For the case of one piezoceramic layer, design the optimum thickness and the optimum electrode arrangement of three electrodes by applying Eqs. (23) and (24).

(2) Step-2: For the case of multiple piezoceramic layers, design the optimum total thickness of the piezoceramic layers when the thicknesses of all piezoceramic layers are equal and the best arrangement of three electrodes obtained in the previous paper ${ }^{(10)}$ is attached to all piezoceramic layers.

(3) Step-3: For the case of multiple piezoceramic layers of the determined optimum thickness, design the optimum electrode arrangement of three electrodes on the top 
piezoceramic layer by applying Eqs. (25), in which case the optimum arrangement determined in Step-1 is attached to the other piezoceramic layers.

Applying this efficient optimum design method to a composite disk with four piezoceramic layers, the optimum total thickness of the piezoceramic layers and the electrode arrangement on the top piezoceramic layer are determined and the obtained numerical results are shown in Table 10. It is seen from the table that the maximum thermal stress is suppressed by $38.31 \%$ and it is the highest suppression ratio among the numerical results derived in this study. Compared to the numerical results obtained in the previous paper ${ }^{(10)}$, the suppression ratio increases from $33.7 \%$ to $38.3 \%$.

Table 10 Numerical results for a composite disk with four piezoceramic layers by utilizing the efficient design method $\left(N=4, \overline{c_{i}}=0.00625, M_{i}=3\right)$

\begin{tabular}{|c|c|c|c|c|c|c|c|c|c|c|}
\hline$i$ & $\bar{w}_{i 1}$ & $\bar{w}_{i 2}$ & $\bar{w}_{i 3}$ & $\bar{q}_{i 1}$ & $\bar{q}_{i 2}$ & $\bar{q}_{i 3}$ & $\bar{V}_{i 1} \times 10^{3}$ & $\bar{V}_{i 2} \times 10^{3}$ & $\bar{V}_{i 3} \times 10^{3}$ & $R[\%]$ \\
\hline 1 & 0.325 & 0.050 & 0.375 & 0.000 & 0.025 & 0.225 & 0.2030 & 0.1343 & -0.0738 & \\
\cline { 1 - 8 } 2 & 0.325 & 0.050 & 0.375 & 0.000 & 0.025 & 0.225 & 0.1684 & 0.2134 & -0.0794 & \multirow{3}{*}{38.31} \\
\hline 3 & 0.325 & 0.050 & 0.375 & 0.000 & 0.025 & 0.225 & 0.0876 & 0.1508 & -0.0653 & \\
\cline { 1 - 7 } & 0.125 & 0.125 & 0.225 & 0.000 & 0.200 & 0.325 & -0.1464 & -0.0730 & 0.1408 & \\
\hline
\end{tabular}

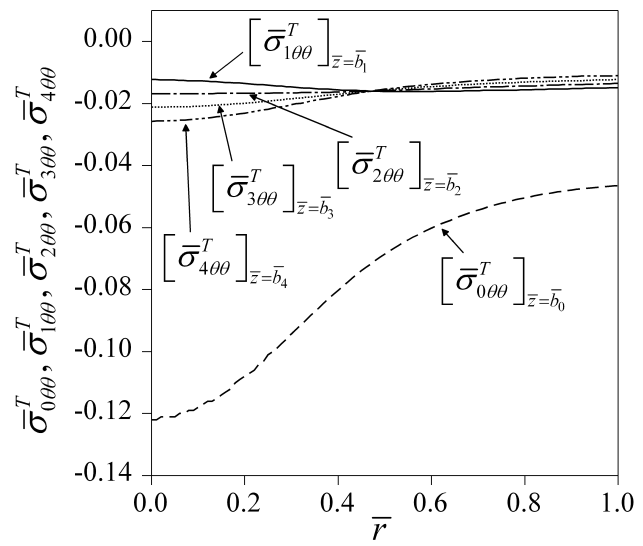

(a) Thermal stresses

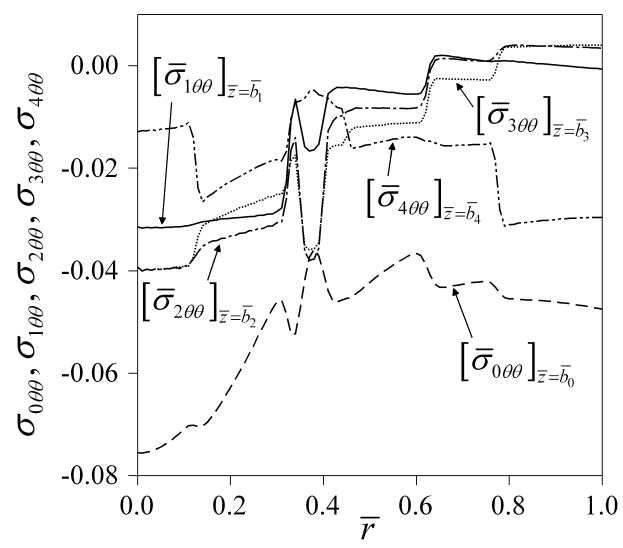

(b) Resultant stresses

Fig. 3 Hoop stress distributions in the optimally designed composite disk with four piezoceramic layers before and after applying determined voltages

Figures 3 illustrate the radial distributions of hoop stresses on the upper surface of each layer of the optimally designed composite disk with four piezoceramic layers before and after applying the determined voltages. These figures show that by applying the determined voltages, the maximum thermal stress yielded in the center of the structural layer surface is reduced from -0.1226 to -0.0756 , whereas the maximum tensile and compressive stresses in the pieozoceramic layers increase, but are less than the stress constraints.

If the old method using only Eqs. (23) and (24) is applied to the design of the arrangement of three electrodes on a piezoceramic layer, about 5,000 data are required. For a composite disk with four piezoceramic layers, the design of the electrode arrangements of three electrodes on all piezoceramic layers needs about 20,000 data even when the design is simply repeated from the first layer to the fourth layer for this once. Employing the efficient optimum design method to the composite disk, it requires 2,000 data at most because only the electrode arrangement on the top piezoceramic layer should be designed. Therefore, the efficient optimum design method greatly reduces the data number required for design of a composite disk with multiple piezoceramic layers. 


\section{Conclusions}

This paper has dealt with a problem of controlling the maximum thermal stress in a composite disk with multiple piezoceramic layers through a piezoelectric actuation. Voltages applied to electrodes on each piezoceramic layer are determined by solving the linear programming problem so that the maximum thermal stress in the structural layer is minimized subject to constraints on the stresses in the piezoceramic layers. The optimal electrode arrangements as well as the optimum layer thicknesses are designed in order to maximize the suppression ratio of the maximum thermal stress. The efficient optimum design method has been developed and thus data required for the design of a composite disk are greatly reduced. A composite disk with four piezoceramic layers has been designed by utilizing the efficient optimum design method, so that the maximum thermal stress in the structural layer is suppressed by $38.3 \%$. Since the highest suppression ratio obtained in the previous study ${ }^{(10)}$ is $33.7 \%$, it increases by $4.6 \%$.

\section{Acknowledgements}

The authors are pleased to acknowledge support in part by a Grant-in-Aid for Scientific Research (No.21560093) from the Japan Society for the Promotion of Science.

\section{References}

(1) Rao, S. S. and Sunar, M., "Piezoelectricity and Its Use in Disturbance Sensing and Control of Flexible Structures: A Survey", ASME Applied Mechanics Reviews, Vol. 47, No. 4 (1994), pp.113-123.

(2) Sunar, M. and Rao, S. S., "Recent Advances in Sensing and Control of Flexible Structures via Piezoelectric Materials Technology", ASME Applied Mechanics Reviews, Vol. 52, No. 1 (1999), pp.1-16.

(3) Irschik, H., "A Review on Static and Dynamic Shape Control of Structures by Piezoelectric Actuation", Engineering Structures, Vol. 24, No. 1 (2002), pp. 5-11.

(4) Tauchert, T. R., Ashida, F., and Noda, N., "Recent Developments in Piezothermoelasticity: Inverse Problems Relevant to Smart Structures," JSME International Journal, Series A, Vol. 42 No. 4 (1999), pp.452-458.

(5) Tauchert, T. R., Ashida, F., Noda, N., Adali, S., and Verijenko, V., "Developments in Thermopiezoelasticity with Relevance to Smart Composite Structures", Composite Structures, Vol. 48, No. 1-3 (2000), pp.31-38.

(6) Tauchert, T. R. and Ashida, F., "Control of Transient Response in Intelligent Piezothermoelastic Structures", Journal of Thermal Stresses, Vol. 26, No. 6 (2003), pp.559-582.

(7) Ashida, F., Tauchert, T. R., Sakata, S., and Yamashita, Y., "Control of Transient Deformation in a Heated Intelligent Composite Disk", Smart Materials and Structures, Vol. 12, No. 5 (2003), pp.825-835.

(8) Ashida, F., Tauchert, T. R., Sakata, S., and Yoshida, S., "Optimum Design of a Piezo-composite Disk for Control of Thermoelastic Displacement Distribution", Journal of Thermal Stresses, Vol. 30, No.6 (2007), pp.559-586.

(9) Ashida, F., Sakata, S., and Matsumoto, K., "Control of Thermal Stress in a Piezo-composite Disk", Journal of Thermal Stresses, Vol. 30, No.9-10 (2007), pp. 1025-1040.

(10) Ashida, F., Sakata, S., and Matsumoto, K., "Structure Design of a Piezoceramic Composite Disk for Control of Thermal Stress", Trans. ASME, Journal of Applied Mechanics, Vol. 75, No.6 (2008), pp. 61009-1-8. 